\title{
Numerical optimization of a bicylindrical resonator impedance: differences and common features between a saxophone resonator and a bicylindrical resonator
}

\author{
Tom Colinot ${ }^{\mathrm{a}}$, Philippe Guillemain ${ }^{\mathrm{a}}$, Jean-Baptiste Doc ${ }^{\mathrm{b}}$, Christophe Vergez $^{\mathrm{a}}$, Michael Jousserand $^{\mathrm{c}}$ \\ a Aix Marseille Univ, CNRS, Centrale Marseille, LMA, Marseille, France \\ b Laboratoire de Mécanique des Structures et des Systèmes couplés, Conservatoire National \\ des Arts et Métiers, Paris, France \\ ${ }^{c}$ Buffet-Crampon, Mantes-la-Ville, France
}

\section{Summary}

This paper explores the analogy between a saxophone resonator and a bicylindrical resonator, sometimes called transverse saxophone or cylindrical saxophone. The dimensions of a bicylindrical resonator are optimized numerically to approximate a saxophone impedance. The target is the impedance measured on an usual saxophone. A classical gradientbased non-linear least-square fit function is used. Several cost functions corresponding to distances to the target impedance are assessed, according to their influence on the optimal geometry. Compromises appear between the frequency regions depending on the cost function. It is shown that the chosen cost functions are differentiable and locally convex. The convexity region contains the initial geometrical dimensions obtained by crude approximation of the first resonance frequency of the target. One optimal geometry is submitted to further analysis using descriptors of the impedance. Its deviations from the target saxophone are put into perspective with the discrepancies between the target saxophone and a saxophone from a different manufacture. Descriptors such as harmonicity or impedance peak ratio set the bicylindrical resonator apart from saxophone resonators, despite a good agreement of the resonance frequencies. Therefore, a reed instrument with a bicylindrical resonator could be tuned to produce the same notes as a saxophone, but due to differences in the intrinsic characteristics of the resonator, it should be considered not as a saxophone but as a distinct instrument.

\section{Introduction}

This work deals with the bicylindrical approximation of a conical geometry, where two cylinders are put in parallel. As a purely academic approximation of a conical instrument such as the saxophone, a "cylindrical saxophone" model permits to obtain analytical results on the produced sound [1,2] and dynamic behavior $[3,4]$. A patent describing bicylindrical resonators to be used for saxophone-like instruments [5] shows that industrial interest exists for such innovative resonator shapes. This paper presents a bicylindrical resonator numerically optimized to replicate the acoustical impedance of an existing saxophone, and compares it to the target saxophone and another "control" saxophone. The goal is to judge whether bicylindrical resonators may be considered as saxophones or not.

Traditionally, instrument makers design new products by trial and error, drawing on empirical knowledge acquired over years of practice. They adjust the manufacturing parameters to maximize the "quality" of successive prototypes in terms of complex criteria involving not only sound characteristics such as intonation and timbre features, but also ergonomics, playability, and feeling of the musician. Given the complexity of this task, as well as the large number of parameters involved, the process is long and requires building several prototypes, amounting to a significant overall cost before the production begins. Numerical optimization may take simple criteria into account to offer geometrical dimensions for new resonators in a quick, repeatable and cheap manner. The optimized resonators would probably have to be fine- 
tuned to satisfy the more complex criteria, but there is hope that overall, the prototyping stage would be accelerated.

From the point of view of an acoustician, the optimization of a musical instrument could, at first, use some criteria pertaining directly to the characteristics of the produced sound, such as the playing frequency. Predicting the sound produced by the instrument for various excitation conditions is possible by numerical synthesis, and has been applied to optimization problems with up to five optimization variables [6]. However, this method is time consuming, hence incompatible with the optimization of dozens of parameters. Consequently, many wind instrument optimization methods adjust the resonance frequencies of the resonator, for instance using an analytic model accounting for small modifications of the bore of a trumpet [7]. Similarly, acoustical considerations were used to adjust iterativelly the positions and dimensions of the holes in a quena to obtain a desired tuning profile [8]. Recent works also propose a method to compute the eigenfrequencies of a variety of multi-cylindrical resonators $[9,10,11]$, with instrument design in mind. For problems with many parameters, computerized optimization strategies are the preferred choice. For example, the complete tone hole geometry of a clarinet was optimized by a gradient descent based on the first and second impedance peaks [12], and the geometry and control of a clarinet model was optimized to reproduce signals obtained with an artificial blowing machine [13]. It is also possible to use the input impedance deduced from a timedomain discretization of the Euler equations in the optimization of a saxophone bore [14]. Some authors take into account the complete input impedance in the cost function, rather than the resonance frequencies alone. This type of objective was applied to trumpets [15] and trombones [16], with Rosenbrock's numerical optimization method [17], and saxophones [18] using the CMA-ES (Evolution Strategy with Covariance Matrix Adaptation) optimization method [19]. In previous work, numerical optimization has mostly served as a tool to adjust or redesign existing instruments, but it can also be seen as a means to explore innovative geometries.

This article presents the optimization of the geometry of a bicylindrical resonator to match the impedance measured on a saxophone, which has a

predominantly conical resonator. These geometries being fundamentally different, the fit cannot be perfect. The acoustical differences between the optimum and the target are analyzed. The optimization is performed numerically, relying on an impedance model of the designed instrument. Objective criteria depending only on linear acoustics considerations are used. This allows to maintain a moderate computational cost. Since our purpose is to compare the bicylindrical resonator with existing saxophones, the target of the optimization relies on impedances measured on a professional alto saxophone (see 2.1). The impedance is measured for several fingerings of the first register, the optimization is performed for these fingerings. By choice, the optimization is limited to a given frequency range: the impedance of the bicylindrical resonator is fitted to the target impedance between 70 and $1200 \mathrm{~Hz}$, which contains the main impedance peaks. The fit is done according to a given norm (see 3.2). The effect of the choice of this norm on the result of the optimization is studied. Moreover, since the optimization algorithm is local, the initial condition is modified to check if the procedure still converges to the same optimum (see 3.3). The influence of each design parameter on the cost function is revealed around the optimum. Finally, the optimized geometry for the example studied here is presented in section 4. The numerical optimization procedure yields the bicylindrical resonator that is as close as possible to a saxophone - considering our criterion and our degrees of freedom. The purpose of this process is to better define the limit of the approximation of a saxophone resonator by a bicylindrical resonator, when it is conducted on several fingerings of the saxophone. The deviations between the impedances of the optimized geometry and the target saxophone are computed, and they are compared with the discrepancies between the target saxophone and a saxophone of another brand.

\section{Input impedance of the sax- ophone and the bicylindrical resonator}

In order to optimize the dimensions of the bicylindrical resonator (see figure 1), it is necessary to use a model giving the impedance of such resonator
152 
based on its geometrical dimensions. The computed impedance is then fitted to the target impedance : the impedance measured on a saxophone, for 16 fingerings of the first register.

\subsection{Saxophone impedance measure- ment: target and control}

Impedance measurements were performed on two saxophones. The first produces the target impedance. The second saxophone, of a different model and different brand, is called the "control saxophone". It serves as reference in the analysis of the difference of characteristics between the bicylindrical resonator resulting from the optimization and the target instrument. This way, we aim to check whether the optimized resonator is as close to the target saxophone as another saxophone. If the differences between two saxophones are of the same order than the differences between the bicylindrical resonator and a saxophone, then the bicylindrical resonator may be considered as a saxophone, at least from the input impedance point of view. The details of this comparison are presented in section 4. The target instrument and the control instrument are commercial models of alto saxophones.

Impedance measurements are carried out using the impedance sensor apparatus developed in [20] on the first register (closed register hole) of the target and control saxophones. All the measurements are carried out in a semi-anechoic room. In total, 16 fingerings of the first register are measured. In written pitch for the alto saxophone, the fingerings range from the low $\mathrm{B} b$ to the $\mathrm{C} \sharp 2$ of the first register - which correspond to the notes Db3 $(138.59 \mathrm{~Hz})$ to $\mathrm{E} 4(329.63 \mathrm{~Hz})$ in concert pitch. The written pitch notation is kept throughout the rest of this paper.

Since the apparatus does not allow impedance measurements of the instrument with its mouthpiece, a cylindrical mouthpiece chamber of typical dimensions (radius $12 \mathrm{~mm}$ and length $L_{m}=60 \mathrm{~mm}$ ) is added in post-treatment, such that the dimensionless target impedance writes

$$
\mathcal{Z}_{\text {tar }}=\frac{Z_{c, m} j \tan \left(k_{m} L_{m}\right)+Z_{\text {mes }}}{Z_{c, m}+Z_{\text {mes }} j \tan \left(k_{m} L_{m}\right)},
$$

where $Z_{\text {mes }}$ is the input impedance measured without the mouthpiece. In order to eliminate the noisy parts of the measurement, the target impedance is truncated at low frequencies, below $70 \mathrm{~Hz}$. To de- crease the computation time of the optimization, the target impedance is also truncated above $1200 \mathrm{~Hz}$. For the fingerings considered, the main impedance peaks fall between 70 and $1200 \mathrm{~Hz}$. Beyond $1200 \mathrm{~Hz}$ the combined effect of the conicity of the resonator and the tone hole network contribute to lowering the impedance peaks. The actual target impedance is discrete vector with 1413 samples, the frequency step between two samples being $0.8 \mathrm{~Hz}$.

\subsection{Impedance of a bicylindrical res- onator}

The bicylindrical resonator, as defined in [21], is composed of a cylindrical mouthpiece (i.e. a mouthpiece with cylindrical chamber) followed by the parallel association of two cylinders (see figure 1). Therefore, the entire instrument's dimensionless input impedance writes

$$
\mathcal{Z}_{\text {des }}=\frac{Z_{c, m} j \tan \left(k_{m} L_{m}\right)+Z_{t s}}{Z_{c, m}+Z_{t s} j \tan \left(k_{m} L_{m}\right)},
$$

where $L_{m}$ is the length of the cylindrical mouthpiece, $Z_{c, m}=\rho c / S_{m}$ is its characteristic impedance depending on its cross section $S_{m}$, the ambient air density $\rho$, the sound velocity $c$. It is worth noting that the parameter $L_{m}$ should be understood as the equivalent length of the chamber of the mouthpiece, not including the length of the reed. $Z_{t s}$ is the input impedance of the parallel association of two cylinders. The wave number $k_{i}$ [22] depends on the equivalent radius $r_{i}$ of each section such that

$$
k_{i}(\omega)=\frac{\omega}{c}-(1+j) 3.10^{-5} \frac{\sqrt{\omega / 2 \pi}}{r_{i}},
$$

where $(i=\{b, m\})$ associates with the long cylinder $b$ or the mouthpiece $m$. The short pipe $a$ is defined by its equivalent radius

$$
r_{e q, a}=\sqrt{S_{a} / \pi},
$$

where $S_{a}$ is the annular cross-section between the inner wall of cylinder $a$ and the outer wall of cylinder $b$ (see figure 1). Since this pipe is ring-shaped, losses are adjusted by a factor $\mu^{\prime}$ corresponding to the ratio of the internal wall surface of the ring $a$ to the internal 


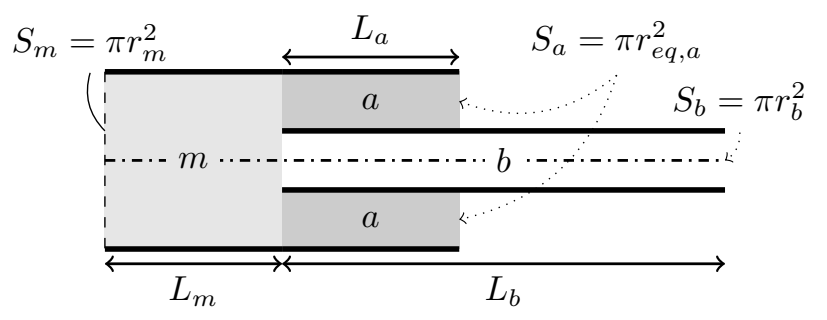

Figure 1: Schematic layout of the optimized resonator : a bicylindrical resonator. Labels: mouthpiece chamber $m$, short cylinder $a$ and long cylinder $b$.

wall surface of a cylinder of radius $r_{e q, a}$

$$
\begin{array}{r}
k_{a}(\omega)=\frac{\omega}{c}-\mu^{\prime}(1+j) 3.10^{-5} \frac{\sqrt{\omega / 2 \pi}}{r_{e q, a}}, \\
\mu^{\prime}=\frac{\sqrt{r_{e q, a}^{2}+\left(r_{b}+e\right)^{2}}+r_{b}+e}{r_{e q, a}},
\end{array}
$$

$e$ being the thickness of the wall of cylinder $b$, fixed at $1 \mathrm{~mm}$ for the rest of the article.

The impedance $Z_{t s}$ of the parallel association of cylinders seen from the end of the mouthpiece $L_{m}$ is written as

$$
\begin{aligned}
Z_{t s}=Z_{c, m}( & \frac{Z_{c, a}+Z_{r, a} j \tan \left(k L_{a}\right)}{Z_{c, a} j \tan \left(k_{a} L_{a}\right)+Z_{r, a}} \\
& \left.+\frac{Z_{c, b}+Z_{r, b} j \tan \left(k_{b} L_{b}\right)}{Z_{c, b} j \tan \left(k_{b} L_{b}\right)+Z_{r, b}}\right)^{-1}
\end{aligned}
$$

where $L_{i}$ is the length of each cylinder $(i=\{a, b\})$, $Z_{c, i}=\rho c / S_{i}$ the characteristic impedance of cylinders $a$ and $b$ and $Z_{r, i}$ the radiating impedance (according to [23]) on the output of the equivalent unflanged cylinder $i$ such that

$$
Z_{r, i}=Z_{c, i}\left(j k_{i} \Delta \ell_{i}+\frac{1}{4}\left(k_{i} r_{i}\right)^{2}\right)
$$

In this expression, the length correction $\Delta \ell_{i}$ is taken as $0.6133 r_{i}$, because both cylinders are assumed unflanged and the influence of their thickness at output is ignored. The influence of the long cylinder on the radiation of the short one is neglected, which corresponds to a plane-wave approximation. A comparison with a flanged impedance radiation model [24] for the output of the short cylinder yields almost no difference in the considered frequency range. These impedance models of the bicylindrical resonator are validated by comparison with impedance measure- ment carried out on a bicylindrical resonator prototype in [21].

\subsection{Initial geometrical parameters of the optimization}

For the optimization on the 16 notes of the first register of the designed instrument, the 20 varying parameters of the model are

$$
X=\left\{L_{b 1}, \ldots, L_{b 16}, r_{b}, L_{a}, r_{e q, a}, L_{m}\right\}
$$

See figure 1 for a schematic representation of the geometry. $L_{b n}$ corresponds to the length of the longest cylinder for the $n^{\text {th }}$ fingering. This definition corresponds to a low frequency approximation of the tone holes: each fingering is represented by an effective length, that can be interpreted as the distance from the input of the instrument to the first open tone hole for this fingering. In this approximation, the effect of the other open tone holes and their interactions are ignored. Note that as a refinement, the optimization procedure is conducted taking into account the effect of the tone hole network in appendix B. The other parameters $r_{b}, L_{a}, r_{e q, a}, L_{m}$ are geometrical dimensions of the designed instruments that cannot be changed between notes.

At the start of the optimization, the parameters of the bicylindrical resonator are assigned initial values. For a gradient-based optimization procedure like the one used in this work (see section 3), the optimized geometry is obtained by adjusting this initial geometry. The initial set of parameters should be chosen in a vicinity of the optimal parameters. Here, based on our knowledge of the characteristics of a bicylindrical resonator, it is possible to suggest an initial geometry that is a coarse approximation of the target, as explained below.

Among the twenty parameters to initialize, two are chosen based on the geometrical dimensions of an alto saxophone: the initial length of the short cylinder $L_{a}^{0}$ is set at $200 \mathrm{~mm}$, which corresponds to the missing length of the top of the conical resonator, and the initial mouthpiece length $L_{m}^{0}$ is set at $10 \mathrm{~mm}$. The length of the long cylinder is based on the first-order approximation of the first resonance frequency for the bicylindrical resonator, taking into account the length corrections due to the radiation impedance. $L_{b, n}^{0}$ (for

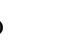


all fingerings $n \in\{1,16\})$ is set so that

$$
\frac{c}{2\left(L_{b, n}^{0}+L_{a}^{0}+2 L_{m}^{0}+0.6133 r_{e q, a}^{0}+0.6133 r_{b}^{0}\right)}=f_{n}
$$

where $f_{n}$ is the frequency of the $n^{\text {th }}$ note based on the tempered scale. The initial cross sections of the tubes are taken equal, such that their initial equivalent radii are $r_{e q, a}^{0}=r_{b}^{0}=4.3 \mathrm{~mm}$ (see figure 1). The total input section of the initial geometry is the same as the one of the measured instrument (see 2.1). The choice of this initial geometry is not critical to the convergence of the optimization algorithm, as the robustness test of subsection 3.3 shows.

\section{Optimization procedure}

In this section, a set of geometrical parameters for the designed instrument is provided by a numerical fit of its input impedance to a target impedance, using a gradient-based, nonlinear least squares optimization procedure.

\subsection{Optimization method}

The optimization is performed through a gradientbased approach (trust-region reflective algorithm), using the lsqnonlin function from the Matlab Optimization toolbox. This function implements nonlinear least-square curve fitting with a convenient interface. The algorithm used is trust-region-reflective [25]. This algorithm is chosen because it allows bounds on the parameters: in our case, all parameters must remain positive. It is inherently local, which means it may converge to different local minima depending on initial conditions. Due to the size of the problem, the maximal number of evaluations of the cost function is set at 20000 and the maximal number of iterations at 1000. Stopping criteria are based on thresholds: the algorithm stops under a chosen variation of cost function per step, a chosen step length, a chosen optimality descriptor value or a chosen cost function value. In all the optimization procedures presented here, the algorithm stops because the variation of the cost function value at a given step is too low. This threshold may be lowered (from the default $10^{-6}$ to $10^{-12}$ ) to give very precise value of the optimal parameters. Section 3.3 shows that the choice of initial conditions is not critical for the case at hand, and the optimum found is valid over a large region of the parameter space. The convergence properties of this algorithm also depend on the derivability properties of the cost function [26]: the convergence of the algorithm is proven (with some assumptions on the problem) for a twice continuously differentiable cost function. This property is verified by the cost functions used in this work (see 3.2 and appendix A). In addition, the solver is rather fast: one optimization procedure lasts about 10 seconds on a laptop computer.

\subsection{Choice of the cost function}

In this work, it is decided to use a cost function taking into account the complete input impedance, over a given frequency range. This choice is motivated by the lack of a priori knowledge on the relative importance of specific impedance descriptors, such as resonance frequency and peak height, for an unusual type of resonator. Still, an assumption is made that highamplitude impedance peaks play a crucial role in the sound production (see for instance [27]). Therefore, we investigate norms under the form

$$
J_{p}(\omega, X)=|| \mathcal{Z}_{\text {des }}(\omega, X)|-| \mathcal{Z}_{\text {tar }}(\omega)||^{p}
$$

where $p$ is an integer, and $\mathcal{Z}_{\text {tar }}$ and $\mathcal{Z}_{\text {des }}$ are respectively the impedance of the target and designed instruments. The notation $X$ stands for the vector of optimization variables. Another motivation for choosing this type of function is that a straightforward mathematical expression allows for easy demonstration of properties of the cost function, like derivability. For $\mathcal{Z}_{\text {des }} \neq 0$ (which is the case for $\omega \neq 0$ ), these cost functions are at least twice continuously differentiable with respect to the optimization variables (see appendix A), which is beneficial to the convergence properties of the optimization algorithm [26]. Figure 2 displays two cost functions (defined by eq. (11)) for $p=2$ and $p=5$. It can be seen on this figure that, as expected, the highest exponent gives more importance to the impedance peaks relative to the troughs. Indeed, a high exponent makes the cost function tend towards a infinite norm.

The optimization algorithm minimizes the sum of the cost function values over the whole frequency range for every considered fingerings: the cost func- 


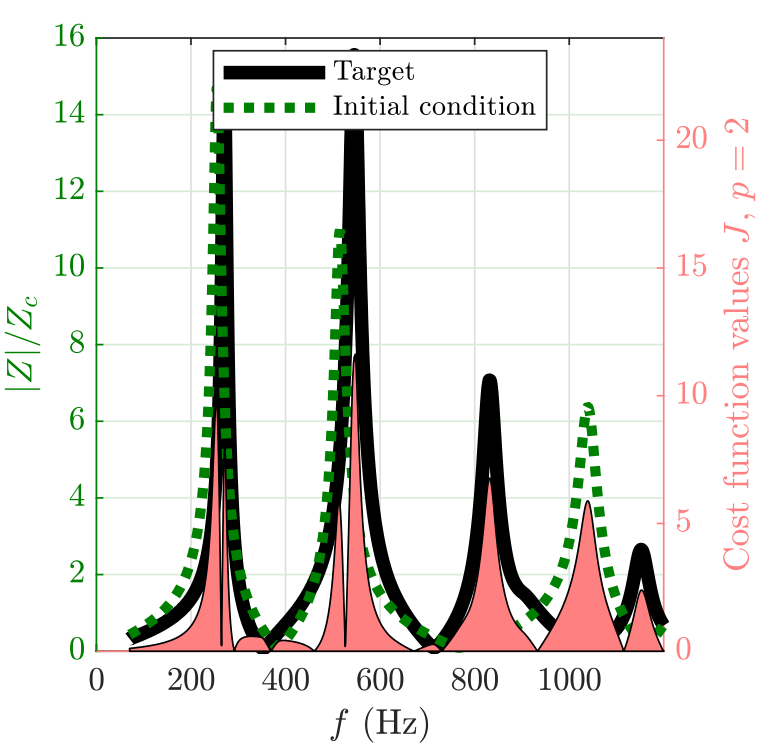

(a)

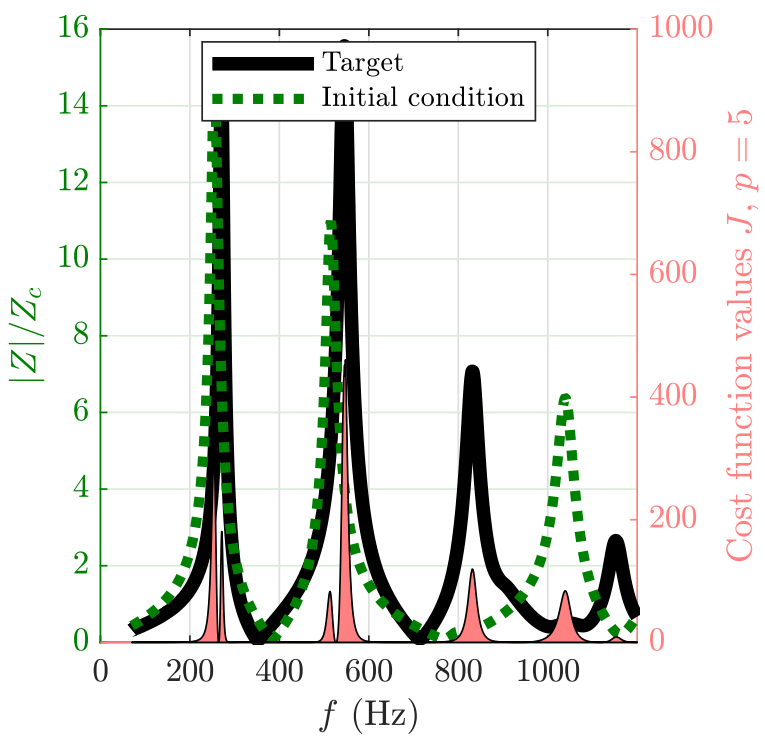

(b)

Figure 2: Comparison before optimization, between the target impedance (solid line) and initial impedance for the bicylindrical resonator (dashed line) for the A fingering. The area is cost function eq. 11 between the two impedances: (a) with $p=2$; (b) with $p=5$. Note that the magnitude of the cost functions values (right axes) is very different between (a) and (b).

tion that is effectively minimized is

$$
J_{p}^{f u l l}(X)=\sum_{n=1}^{16} \sum_{\omega=\omega_{\min }}^{\omega_{\max }}|| \mathcal{Z}_{\text {des }, n}(\omega, X)|-| \mathcal{Z}_{\text {tar }, n}(\omega)||^{p}
$$

where $\mathcal{Z}_{d e s, n}$ and $\mathcal{Z}_{\text {tar }, n}$ are the impedances of the $n^{\text {th }}$ fingering, respectively for the bicylindrical resonator and the target instrument, and $\omega_{\min }=2 \pi \times 70 \mathrm{rad} . \mathrm{s}^{-1}$ and $\omega_{\max }=2 \pi \times 1200 \mathrm{rad} . \mathrm{s}^{-1}$ are the angular frequencies at which the impedances are truncated.

In order to explore the influence of the exponent $p$ on the optimal geometry, several optimization procedures are launched using the 1sqnonlin function, the only difference being the exponent $p$ of the cost function. Five values of $p$ are tested: 1,2,3,5 and 10. The optimal sets of geometrical parameters are slightly different. As expected, the highest $p$ exponents give a more accurate fit of the impedance peaks with the greatest modulus, at the expense of the lowest. The ratios between the frequency of the impedance peaks are conserved, which could be expected since the bicylindrical resonator has few geometrical degrees of freedom. For the target instrument, the first impedance peak, which corresponds to the first register, is lower than the next for the 12 first fingerings (from low $\mathrm{B} b$ to high $\mathrm{B} b$ ). The value $p=1$ is set aside because of the differentiability issue it entails and because the impedance minima are of lesser importance than the maxima. Therefore, the exponent $p=2$ is chosen for the cost function so as not to reduce the importance of the first peak in the optimization too much.

\subsection{Robustness of the optimization procedure}

The chosen method is a local optimization procedure. As such, its result depends on the initial conditions, so we seek to further qualify the validity of the optimum, particularly its robustness to a change of initial geometry. As announced in subsection 3.2, the exponent in the cost function (eq. 11) is $p=2$ from now on.

As a preliminary study, the optimization procedure is tested using a temporary target: a simulated impedance for a bicylindrical resonator with known geometry (the geometry of the optimum presented in section 4). The result of this optimization can then be assessed, by comparing it to the known geometry of the temporary target. A test is performed in which the optimization procedure starts with 50 different initial geometries placed around the geometry of the temporary target. Each parameter is placed at a certain initial distance from its value for the temporary target, yielding a set of extreme initial geometries. 
The possible distances are $\pm 80 \mathrm{~mm}$ for each length $L_{b, n}, \pm 30 \mathrm{~mm}$ for the length of the short cylinder $L_{a},[-2,+5] \mathrm{mm}$ for the radius $r_{b}$ and the equivalent radius $r_{e q, a}$, and $[-10,+30] \mathrm{mm}$ for the mouthpiece chamber length $L_{m}$. With these extreme initial conditions, the algorithm converges to optimal dimensions within $10^{-3} \mathrm{~mm}$ of the dimensions of the temporary target. For this controlled problem, this procedure gives the order of magnitude of the size of basin where the optimized geometry converges to the correct optimum.

We now apply a similar method to studying the main optimization problem: optimizing the bicylindrical resonator to fit a target impedance measured on a saxophone. In this case, 50 initial geometries are generated, each parameter within a certain range of the value assigned to it in section 2.3. This range is set as $\pm 10 \%$ for each $L_{b, n}, \pm 30 \%$ for $L_{a}$ and $L_{m}, \pm 50 \%$ for $r_{b}$ and $r_{e q, a}$. The size of the range is inspired by the preliminary test with the temporary bicylindrical target and adapted to fill the convergence basin.

With 50 different initial geometries, the optimization converges every time to similar optimal geometries: less than $0.002 \%$ of variation for each optimal parameter, except for the optimal mouthpiece lengths $L_{m}$ which has a $0.01 \%$ spread (less than $2 \mu \mathrm{m}$ ). This larger spread on the parameter $L_{m}$ can be explained by looking at its influence on the cost function (figure 6 , detailed below). The dispersion on optimal value is due to the stopping criterion of the optimization procedure and can be reduced by restricting the conditions under which the algorithm stops. One of the conclusion that can be drawn from this result is that even though the optimization procedure is local, the initial geometry is not critical: when it is changed the resulting optimal geometry remains the same.

In order to appreciate the evolution of the cost function, the history plot of its value during the optimization is plotted in figure 3. In this figure, the different fingerings are separated (inner sum in equation (12)). Most of the improvement is accomplished during the first five iterations. During the rest of the optimization, compromises between fingerings appear. It can be seen that for some fingerings, one of the earlier iterations has a better cost function value than the final iteration. The fit of those fingerings is then degraded to improve the global value of the cost function.

To gain information on the convergence behavior

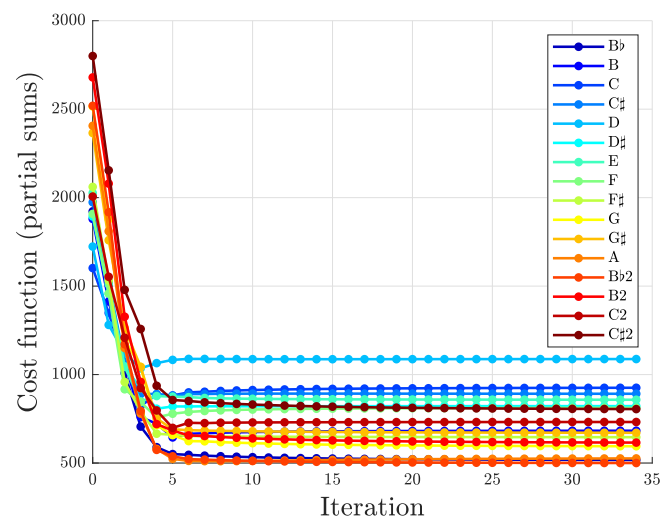

Figure 3: Evolution of the cost function values during the optimization: partial sums over each fingering (inner sum in Eq. 12).

that can be expected from the algorithm, it is useful to study the projection of the cost function around the optimal set of parameters. Here, it is chosen to compute the cost function over the complete frequency range and the 16 fingerings by varying one or two of the parameters around the optimum (all the other parameters are left at their optimal values).

Figure 4 shows the variation of the cost function depending on each length of the long cylinder $L_{b}$, within $100 \mathrm{~mm}$ of the initial lengths. All the other parameters are fixed at their optimal values. The cost function appears locally convex, and the optimum corresponds to the minimum of the cost function in the plotted range for each lengths: choosing any set of initial lengths $L_{b}$ in a $100 \mathrm{~mm}$ range from the optimum appears viable to obtain convergence. Initial points used in the robustness test are between brackets on figure 4 . They are all in the convexity region according to the represented projections. This is coherent with the algorithm converging every time.

The projection of the cost function space on the parameters $r_{b}$ and $r_{e q, a}$ is displayed on figure 5. It may be noted, on figures 5 and 6 , the initial conditions are not on the surface representing the projection of the cost function around the optimum. This is because all the parameters of the initial conditions differ from their optimal value, whereas the surface is constructed by varying only two parameters. Once again, the cost function appears convex, although there is a slope inversion for very small values of the radius $r_{b}$. It can be noted that the configuration where the two radii are equal seems privileged (a local minimum follows the 


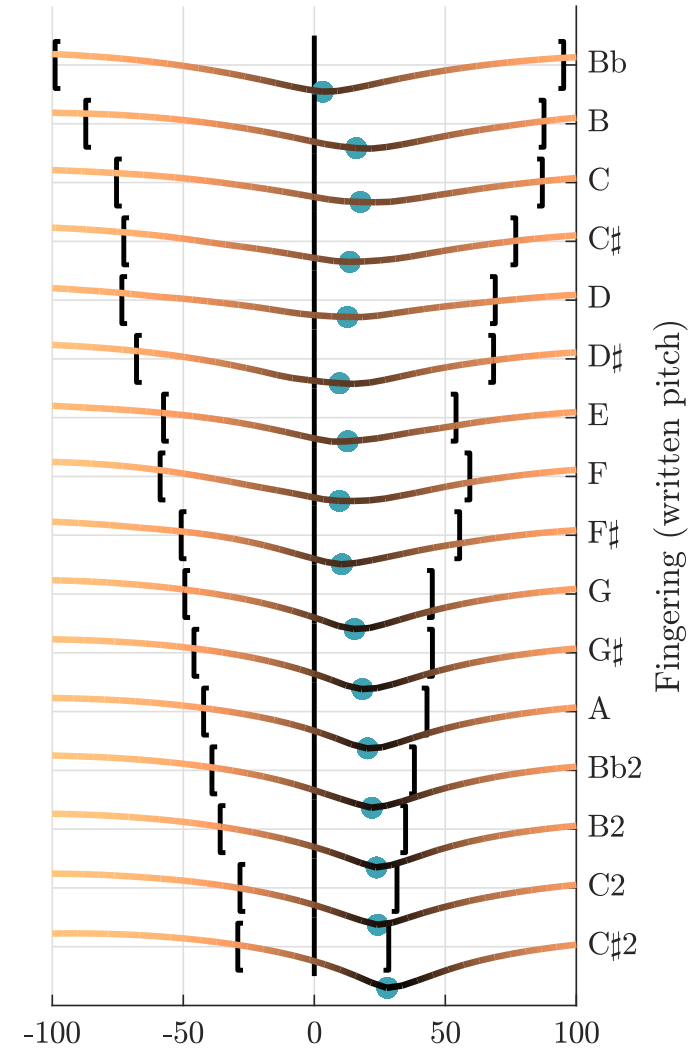

$L_{b}$ : discrepancy from initial value, $\mathrm{mm}$

Figure 4: Variation of the cost function of Eq. (12) (solid lines) depending on each parameter $L_{b}$, around the optimum obtained for $p=2$ in Eq. 11 (light dot). The displayed cost function values are normalized. All lengths are displayed with respect to the initial values (vertical black line). The brackets stand for the minimal and maximal initial lengths used in the robustness test.

main diagonal on the figure). This is the configuration of the usual cylindrical saxophone approximation [1].

Figure 6 displays the projection along the length of the short cylinder $L_{a}$ and the length of the mouthpiece $L_{m}$. There, two features may be noted: there is a slope inversion for lengths of the short cylinder above $L_{a}=230 \mathrm{~mm}$ and below $L_{a}=50 \mathrm{~mm}$, and the length of the mouthpiece $L_{m}$ appears to have small influence on the cost function value. This explains the larger dispersion in optimal mouthpiece lengths: a change in the parameter $L_{m}$ amounts to a very small modification of the cost function value.

It can be seen on the figures 4,5 and 6 that the cost function appears continuously differentiable, as announced in subsection 3.2. This is one of the nec-

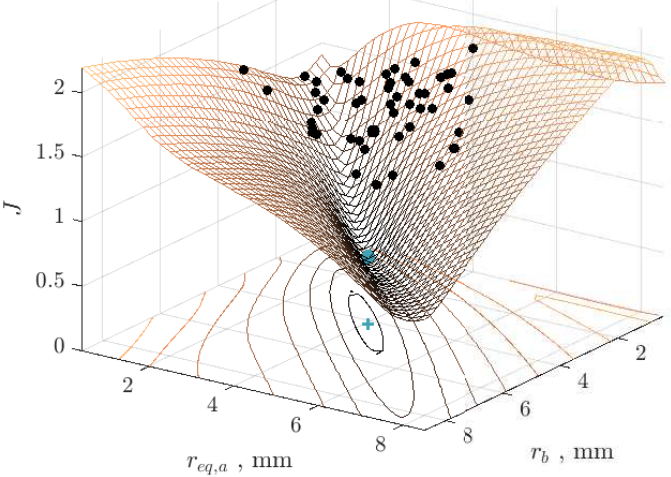

Figure 5: Variation of the cost function of Eq. (12) (mesh) depending on the radii of the two cylinders $r_{b}$ and $r_{e q, a}$ around the optimum obtained for $p=2$ in Eq. 11 (clear dot). Black dots: initial conditions in the robustness test.

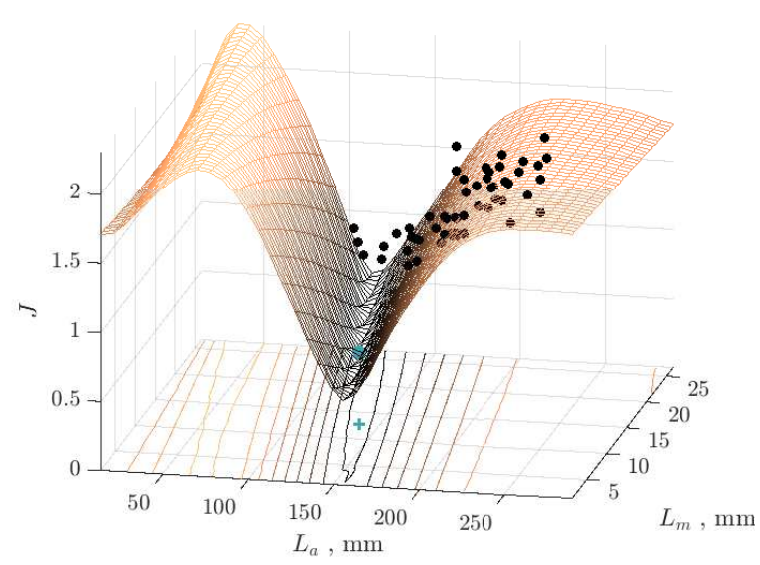

Figure 6: Variation of the cost function of Eq. (12) (mesh) depending on the lengths of the short cylinder $L_{a}$ and the mouthpiece $L_{m}$ around the optimum obtained for $p=2$ in Eq. (12) (clear dots). Black dots: initial conditions in the robustness test.

essary hypotheses in the proof of the convergence of the trust-region reflective algorithm of the lsqnonlin function. Overall, this study on the profile of the cost function near the optimum contributes to justifying the use of a local, gradient-based optimization method. 
Colinot et al., p. 9

The optimization procedure yields geometrical dimensions for the designed instrument, summarized in table 1. Several comments can be made on the proposed values of the geometrical parameters, notably in relation to the dimensions of the target instrument. The length of the instrument $L_{b}$ corresponds to the approximate length of the bore of an alto saxophone, ranging from $1000 \mathrm{~mm}$ to under $300 \mathrm{~mm}$. In the coaxial configuration of the bicylindrical resonator (see figure 1) where the short cylinder is around the long cylinder, the total input radius of the optimized resonator is

$$
r_{m}=\sqrt{S_{m} / \pi}=\sqrt{\frac{\pi r_{e q, a}^{2}+\pi\left(r_{b}+e\right)^{2}}{\pi}}=6.6 \mathrm{~mm} .
$$

It is very close to the input radius of the target instrument, $6.0 \mathrm{~mm}$. However, the optimal mouthpiece is shorter than the mouthpiece added to the impedance measurements of the target instrument (12.5 mm versus $60 \mathrm{~mm}$ ). This is consistent with the usual formulation of the cylindrical saxophone approximation, where a complete conical instrument including its mouthpiece is replaced by two parallel cylinders without any mouthpiece [4]. This suggests that the mouthpiece chamber should be as short as possible, which is possible in the coaxial configuration (see figure 1).

For further analysis of the optimum geometry, it is necessary to consider the input impedance of the designed instrument, computed from (2). For the sake of clarity, among the total of 16 fingerings in the opti-

\begin{tabular}{|l|c|c|c|}
\hline Long & radius & $r_{b}$ & $4.2 \mathrm{~mm}$ \\
cylinder & lengths & $L_{b}$ & {$[323.6 ; 1016.9] \mathrm{mm}$} \\
\hline Short & Eq. radius & $r_{e q, a}$ & $4.1 \mathrm{~mm}$ \\
cylinder & length & $L_{a}$ & $138.2 \mathrm{~mm}$ \\
\hline Mouthpiece & length & $L_{m}$ & $12.5 \mathrm{~mm}$ \\
\hline
\end{tabular}

Table 1: Optimized geometrical parameters of the designed bicylindrical resonator.

mization procedure, 2 fingerings are displayed in figure 7. They correspond to the low $\mathrm{B}$ and the $\mathrm{A}$ in written pitch, or $D_{3}=146.83 \mathrm{~Hz}$ and $C_{4}=261.63 \mathrm{~Hz}$ in concert pitch. Even though the complete display of the impedance holds a quantity of information too large to be interpreted clearly, it is natural to look at it first in this context: the optimization procedure aims to match the impedance curves themselves (see Eq. (12)) and does not rely on impedance descriptors. In the frequency range where the optimization is performed, from $\omega_{\min } /(2 \pi)=70 \mathrm{~Hz}$ to $\omega_{\max } /(2 \pi)=1200 \mathrm{~Hz}$, the impedance corresponding to the optimum shows good qualitative agreement with the target. The impedance peaks are slightly higher for the target. This phenomenon can be related to the difference in the geometry of the two instruments: the optimized geometry being composed only of cylinders, the losses and radiation mechanisms differ from those encountered in the mainly conical resonator of the target instrument. In terms of phase, the impedance of the designed instrument fits that of the target more accurately at the resonances (i.e. when the phase goes from positive to negative) than at the anti-resonances. The chosen cost function (see 3.2) appears to have emphasized the importance of these peaks in the optimization strategy. However, the bicylindrical resonator shows additional resonances in high frequency - between $1200 \mathrm{~Hz}$ and $2200 \mathrm{~Hz}-$ above the optimized region. This second group of peaks is inherent to the bicylindrical geometry, but it is worth noting that an adequate tone hole network could attenuate these peaks, by introducing a cutoff frequency [28]. In terms of global impedance shape, this phenomenon is the major difference with real saxophones. It is possible that these resonances would affect the production of sound. 

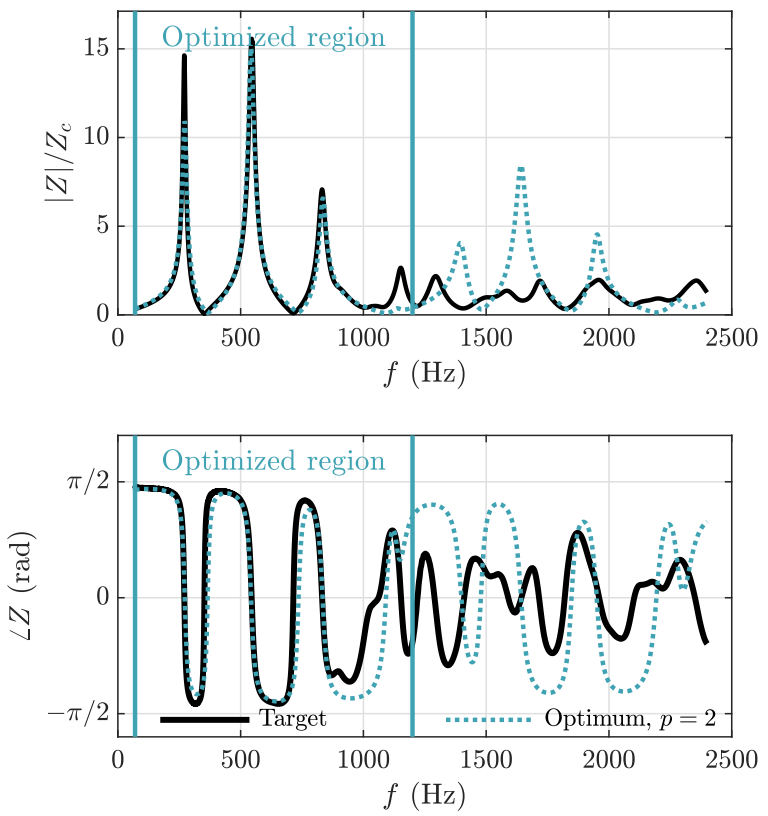

(a)
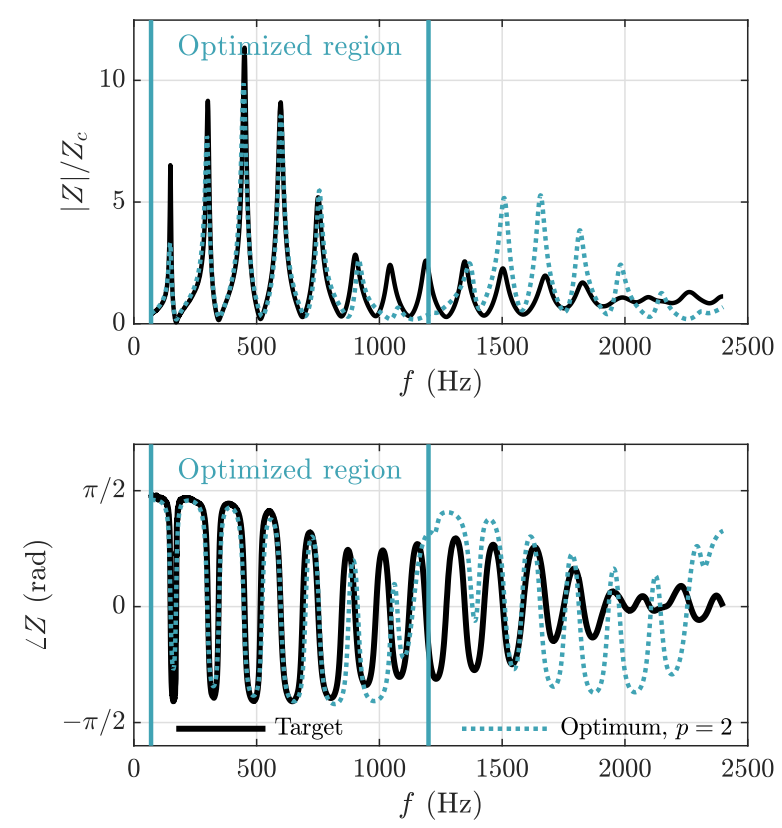

(b)

Figure 7: Comparison between target impedance (solid line) and impedance for the designed bicylindrical geometry (dotted line) for (a) the A fingering and (b) the low B fingering.

\subsection{Comparison between characteris- tics of the impedances}

In order to quantify the difference between the quency of the first resonance, represented by the first impedance peak, that plays a large role in determining the playing frequency for the first register. In practice, these resonance frequencies are detected as the points where the phase passes from positive to negative. Figure 8 compares the frequency of the first impedance peak for every note of the first register of the target instrument and the optimized geometry. The same descriptor is computed for the control saxophone: the differences between the two saxophones serve as references when comparing the optimized geometry to the target. A common reference is taken as the 12-tone tempered scale based on $A_{4}=440 \mathrm{~Hz}$. There is a shared global tendency along the first register: the discrepancy between the resonance frequencies and the reference frequencies becomes larger towards the top of the register. It appears on the figure that the two saxophones (target and control) are closer together than the target and the optimum. There is a good agreement between the optimized resonator and the target for the highest fingerings of the register (high $\mathrm{B} 2, \mathrm{C} 2$ and $\mathrm{C} \sharp 2$ ). This is possibly due to the smaller number of impedance peaks in the frequency range taken into account for the optimization $(70 \mathrm{~Hz}$ to $1200 \mathrm{~Hz}$ ) for the highest fingerings. Indeed, when there is no third or fourth impedance peak in the frequency range, the geometrical degrees of freedom are entirely devoted to fitting the first and second peak. Otherwise, for the rest of the fingerings, a compromise must be made, that leads to a slightly poorer fit of the first peak.

Table 2 summarizes the difference for the first four peaks by averaging the difference over the studied fingerings. Although it is difficult to draw final conclusions from the mean value of an indicator over several fingerings, it is a simple quantitative way to qualify the global difference between target and optimum, and compare it with the difference between the two saxophones. There, we can see that except for the first peak, the average difference between the target and the optimized resonator is similar to the difference between the two real saxophones (target and control). Looking at this average descriptor only, the bicylindrical resonator could be assimilated to a saxophone resonator. However, other descriptors point out the limits of the cylindrical saxophone analogy in terms of impedance characteristics.

Another way to study the resonance frequencies of an instrument is to compare them with its first res- 


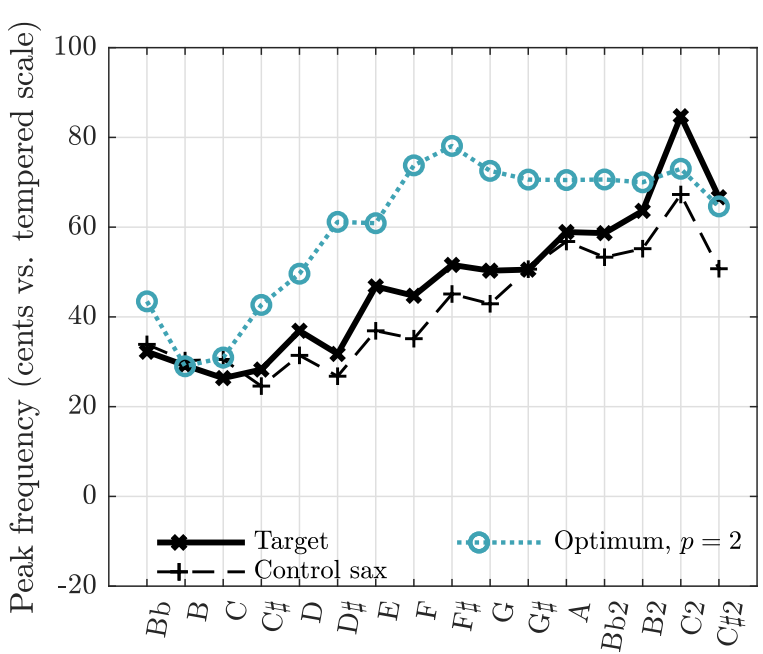

Figure 8: First impedance peak frequency: target (cross, solid), the control saxophone (plus, dashed) and the optimum for the cost function Eq. (12) with $p=2$ (circle, dotted). Discrepancy in cents versus the corresponding notes in the tempered scale.

\begin{tabular}{|l|l|l|}
\hline Instrument & $\begin{array}{l}\text { Optimum, } p=2 \\
\text { vs. Target }\end{array}$ & $\begin{array}{l}\text { Control saxophone } \\
\text { vs. Target }\end{array}$ \\
\hline Peak 1 & +12.5 & -5.61 \\
\hline Peak 2 & -7.47 & -7.96 \\
\hline Peak 3 & +5.77 & +6.18 \\
\hline Peak 4 & +16.5 & +12.1 \\
\hline
\end{tabular}

Table 2: Mean discrepancy to the resonance frequencies of the target, for the optimum $(p=2)$ and the control sax, in cents.

onance frequency. The ratio between the second and the first resonance frequencies has been shown to influence the tone color and tuning of the instrument [27]. A descriptor called harmonicity can be defined

$$
\text { Harmonicity }=100 \times \frac{f_{2}}{2 f_{1}},
$$

expressed in percents, where $f_{1}$ and $f_{2}$ are the first and second resonance frequencies. A global reference when looking at this descriptor is the integer multiples of the first resonance frequency. For instance, if the second resonance corresponds to the octave of the first, the harmonicity for the second peak is worth exactly $100 \%$. Figure 9 shows the harmonicity for the second resonance. The trend along the register clearly differs between the optimum and the two saxophones. It can be noted that the bicylindrical resonator has harmonicity closer to $100 \%$ for the second peak. This is one of the possible characteristics of a bicylindrical resonator compared to a conical one. Doc [29] shows that a few percents of difference on the harmonicity conditions the production of certain regimes, quasi-periodic for example, on a saxophone. The high-frequency resonances that appear with the bicylindrical resonator (see figure 7) may also change the sound production behavior. Therefore, the bicylindrical resonator can be expected to play quite differently from a usual saxophone.

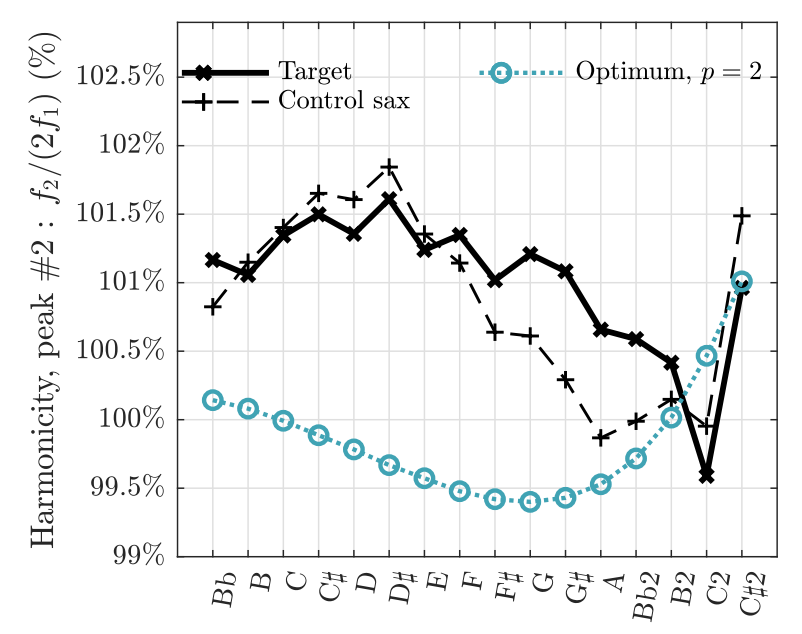

Figure 9: Harmonicity between the second and first impedance peaks for the target (cross, solid), the control saxophone (plus, dashed) and the optimum for the cost function eq (12) with $p=2$ (circle, dotted).

Another indicator of fundamental difference between a usual saxophone and the bicylindrical resonator studied here is the height of the impedance peaks, defined as the impedance modulus at the resonance frequency. As with the harmonicity, the first resonance for each fingering can be taken as reference to study the other, leading to a height ratio of the form

$$
\text { Peak height ratio }=\frac{\left|Z\left(f_{2}\right)\right|}{\left|Z\left(f_{1}\right)\right|} \text {. }
$$

The value of the ratio is very different for the bicylindrical resonator, as shown in figure 10. An analytical and numerical study [30] shows that this may also lead to differences in the sound production characteristics, in particular the ease of playing in the first register. The harmonicity and relative amplitude of the first two peaks may also change the timbre of the instrument, notably by affecting the harmonics of the produced sound. 


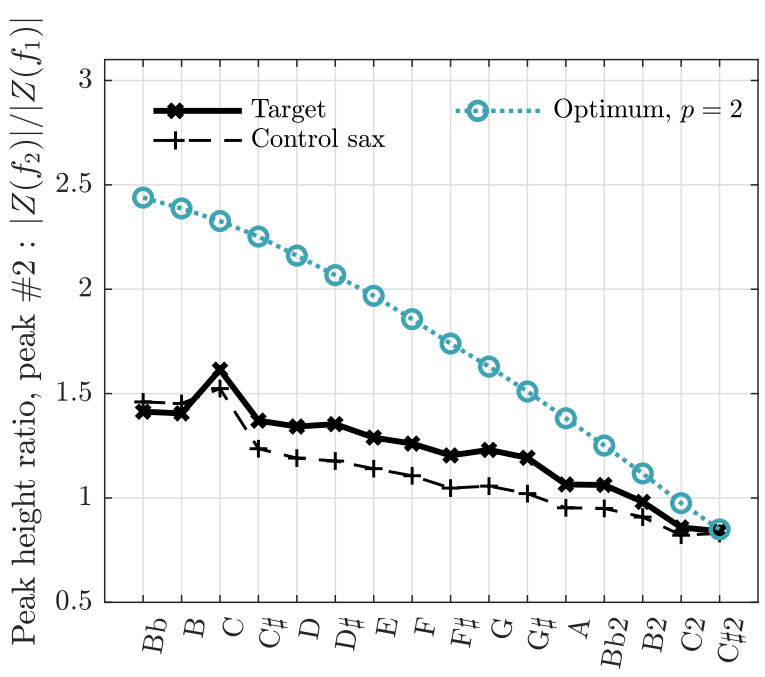

Figure 10: Ratio between the height (modulus of the impedance) of the second and first impedance peak height for the target (cross, solid), the control saxophone (plus, dashed) and the optimum for the cost function Eq. (11) with $p=2$ (circle, dotted).

\section{Conclusion}

The optimization of the bicylindrical resonator to fit impedance measurements performed on an usual saxophone shows that compromises on the optimum are inevitable, to fit certain impedance peaks or others. We have shown that choosing between cost functions allows to emphasize certain parts of the target impedance and control this compromise. Some practical properties of the type of cost functions chosen in this work, such as derivability and local convexity, have been exhibited. In the present case of optimization on a complete instrument with a rather simple geometrical model, a local, least-square method has proven sufficiently robust to initial conditions. The development of original resonators may particularly benefit from optimization procedures, to yield sensible geometrical parameters as a starting point in the design of completely new instruments. In this context, adding geometrical degrees of freedom - for instance the parameters of a tone hole network - would be a way to provide a more precise fit of the target impedance.

Here, the optimized resonator has characteristic trends along the register that are inherent to its cylindrical nature and differ from those of the (conical) target. On the second, third and fourth resonance frequencies alone, the bicylindrical resonator does not differ from the target more than another saxophone does. However, descriptors like harmonicity and peak height ratio show notable differences. The interpretation that can be made from such results is that the bicylindrical resonator can be tuned to produce the same notes as a saxophone, like an oboe may produce the same notes as a saxophone, but intrinsic characteristics of the resonator differ. This means that, even in low frequency, a reed instrument with bicylindrical resonator should probably be envisioned as a new instrument rather than a pure copy of the existing saxophones, although they share some global acoustic features. The bicylindrical geometry requires further study in terms of sound production, to conclude on its similarity with existing saxophones and its viability as a musical instrument.

\section{Acknowledgements}

The authors would like to thank Buffet-Crampon for the loan of the target instrument, and Erik Petersen for his helpful comments. This work has been carried out in the framework of the Labex MEC (ANR-10-LABX-0092) and of the A*MIDEX project (ANR-11-IDEX-0001-02), funded by the Investissements d'Avenir French Government program managed by the French National Research Agency (ANR). This study has been supported by the French ANR LabCom LIAMFI (ANR-16-LCV2-007-01).

\section{A Derivability and derivatives of the cost function}

The cost function defined by Eq. (11) with $p=2$ is twice continuously differentiable, as long as $\left|Z_{\text {des }}(\omega, X)\right| \neq 0$, which is true for strictly positive frequencies. The first order derivative with respect to a given parameter $X_{i}$ writes

$$
\begin{aligned}
\frac{\partial J_{2}(\omega, X)}{\partial X_{i}}= & 2 \mathcal{Z}_{\text {des }}(\omega, X) \frac{\partial \mathcal{Z}_{\text {des }}(\omega, X)}{\partial X_{i}} \\
& \times \frac{\left|\mathcal{Z}_{\text {des }}(\omega, X)\right|-\left|\mathcal{Z}_{\text {tar }}(\omega)\right|}{\left|\mathcal{Z}_{\text {des }}(\omega, X)\right|}
\end{aligned}
$$

where the derivative of the impedance $\mathcal{Z}_{\text {des }}$ with respect to each parameter can be computed from Eq. (2) and exists for nonzero values of the geometrical parameters. The expression in Eq. (16) may be differentiated a second time with respect to a geometri- 
cal parameter, leading to a continuous function, still under the assumption $\left|\mathcal{Z}_{\text {des }}(\omega, X)\right| \neq 0$.

\section{B Optimization of a bicylindri- cal resonator with tone holes}

As a refinement, the impedance model may be modified to include tone holes. An optimization is performed using this model, with tone holes whose radii are identical and fixed at half the radius of the long cylinder. The number of optimization parameters is the same as in the case without tone holes : 1 total length of the main cylinder, corresponding to the lowest note, 15 positions of tone holes (one for each of the other fingerings), the radius of the longest cylinder $r_{b}$, the equivalent radius $r_{e q, a}$ and length $L_{a}$ of the short cylinder and the mouthpiece length $L_{m}$. The optimization is significantly longer (by a factor of 10) due to the added complexity of the impedance model, but the optimum is very close in terms of impedance. Figure 11 shows the comparison between the target and the two optimums. The closeness of the impedances may be explained by the fact that the tone-hole network has a high frequency effect. On figure 11 the impedances start to differ at about $2000 \mathrm{~Hz}$, which is beyond the frequency range taken into account in the optimization. A calculation of the associated cutoff frequency, as the Helmhotz frequency of the resonators formed by each association of a tone hole and the pipe section underneath, yields results between $3.4 \mathrm{kHz}$ and $5 \mathrm{kHz}$, well above the frequency of the main impedance peaks.

\section{References}

[1] A. H. Benade, "Equivalent circuits for conical waveguides," The Journal of the Acoustical Society of America, vol. 83, no. 5, pp. 1764-1769, 1988.

[2] S. Ollivier, J.-P. Dalmont, and J. Kergomard, "Idealized models of reed woodwinds. Part I: Analogy with the bowed string," Acta acustica united with acustica, vol. 90, no. 6, pp. 11921203, 2004.

[3] S. Ollivier, J. Kergomard, and J.-P. Dalmont, "Idealized models of reed woodwinds. Part II: On the stability of "two-step" oscillations," Acta
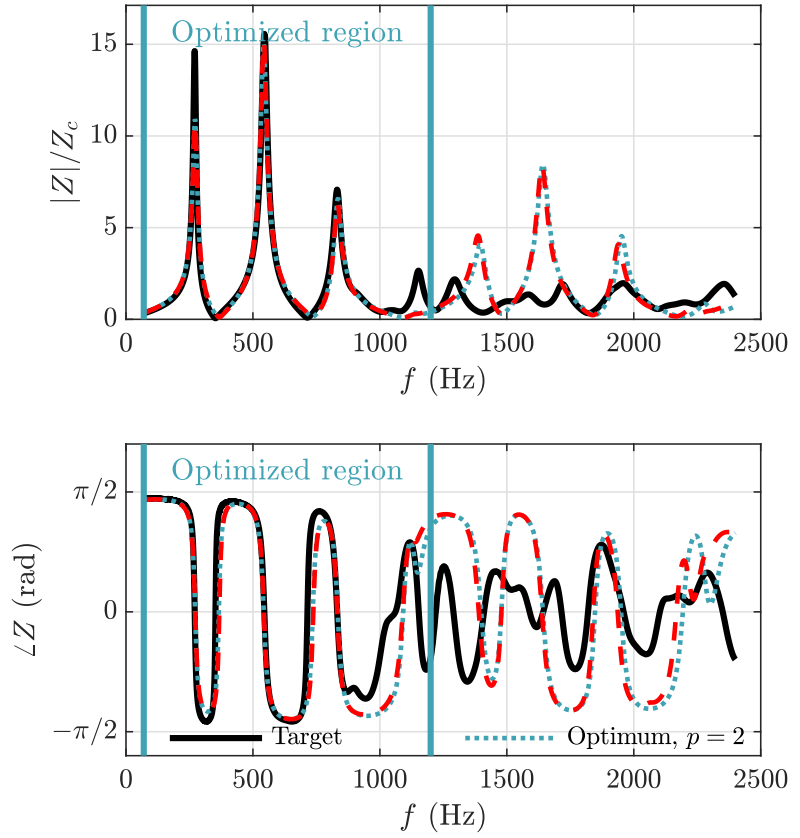

(a)
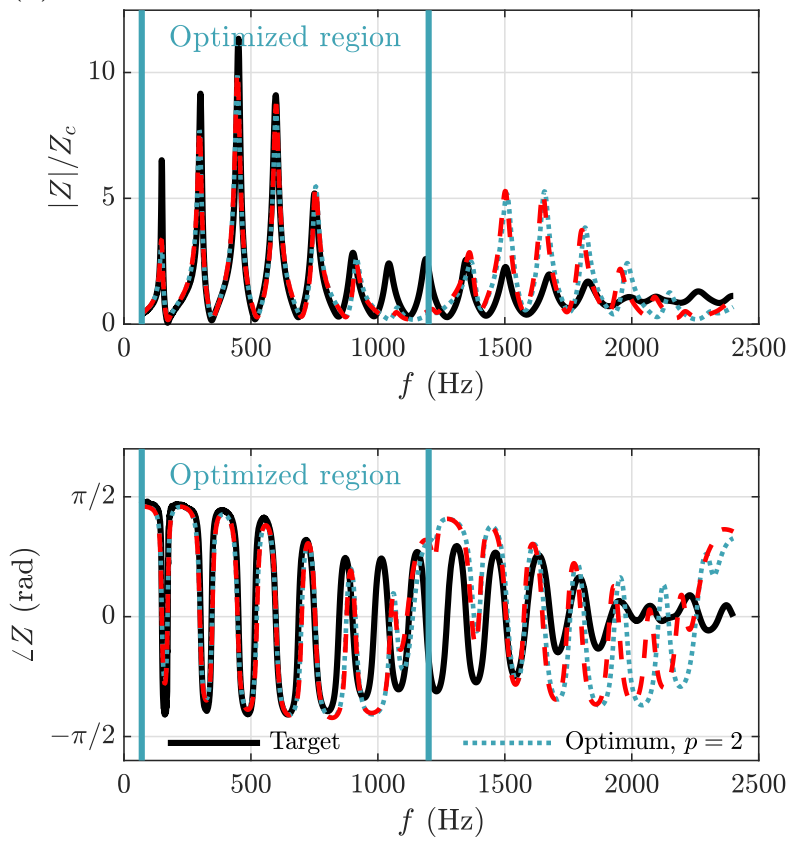

(b)

Figure 11: Comparison between target impedance (solid line), impedance for the optimized bicylindrical resonator without tone holes (blue dotted line) and with toneholes (red dashed line), for (a) the A fingering and (b) the low B fingering.

acustica united with acustica, vol. 91, no. 1, ${ }_{719}$ pp. 166-179, 2005.

[4] J.-P. Dalmont, J. Gilbert, and J. Kergomard, "Reed instruments, from small to large amplitude periodic oscillations and the Helmholtz mo- 
tion analogy," Acta Acustica united with Acustica, vol. 86, no. 4, pp. 671-684, 2000.

[5] H. Masuda and Y. Suenaga, "Pipe structure of wind instrument," Feb. 9 2011. US Patent App. 13/023,793.

[6] R. Tournemenne, J.-F. Petiot, B. Talgorn, M. Kokkolaras, and J. Gilbert, "Brass instruments design using physics-based sound simulation models and surrogate-assisted derivativefree optimization," Journal of Mechanical Design, vol. 139, no. 4, p. 041401, 2017.

[7] C. A. Macaluso and J.-P. Dalmont, "Trumpet with near-perfect harmonicity: Design and acoustic results," The Journal of the Acoustical Society of America, vol. 129, no. 1, pp. 404-414, 2011.

[8] C. Vauthrin, B. Fabre, and P. de la Cuadra, "The design of a chromatic quena: How can linear acoustics help ?," in Stockholm Music Acoustics Conference, 2013.

[9] G. Le Vey, "Graph modelling of musical wind instruments: a method for natural frequencies computation," Acta Acustica united with Acustica, vol. 101, no. 6, pp. 1222-1233, 2015.

[10] J.-P. Dalmont and G. Le Vey, "Discrete acoustical resonators with harmonic eigenfrequencies," Acta Acustica united with Acustica, vol. 103, no. 1, pp. 94-105, 2017.

[11] G. Le Vey, "A mathematical model for air columns in a class of woodwinds," Acta Acustica united with Acustica, vol. 103, no. 4, pp. 676-684, 2017.

[12] D. Noreland, J. Kergomard, F. Laloë, C. Vergez, P. Guillemain, and A. Guilloteau, "The logical clarinet: numerical optimization of the geometry of woodwind instruments," Acta Acustica united with Acustica, vol. 99, no. 4, pp. 615-628, 2013.

[13] V. Chatziioannou, S. Schmutzhard, M. PàmiesVilà, and A. Hofmann, "Investigating clarinet articulation using a physical model and an artificial blowing machine," Acta Acustica united with Acustica, vol. 105, no. 4, pp. 682-694, 2019.
[14] S. Schmutzhard, V. Chatziioannou, and A. Hofmann, "Parameter optimisation of a viscothermal time-domain model for wind instruments," 2017.

[15] W. Kausel, "Optimization of brasswind instruments and its application in bore reconstruction," Journal of New Music Research, vol. 30, no. 1, pp. 69-82, 2001.

[16] A. C. Braden, M. J. Newton, and D. M. Campbell, "Trombone bore optimization based on input impedance targets," The journal of the Acoustical Society of America, vol. 125, no. 4, pp. 2404-2412, 2009.

[17] H. Rosenbrock, "An automatic method for finding the greatest or least value of a function," The Computer Journal, vol. 3, no. 3, pp. 175-184, 1960.

[18] P. Guillemain and J. Kergomard, "Generic resonator models for real-time synthesis of reed and brass instruments," in 6th Forum Acusticum, 2011.

[19] N. Hansen, "The cma evolution strategy: a comparing review," in Towards a new evolutionary computation, pp. 75-102, Springer, 2006.

[20] J.-P. Dalmont and J. C. Le Roux, "A new impedance sensor for wind instruments," The journal of the Acoustical Society of America, vol. 123, no. 5, pp. 3014-3014, 2008.

[21] J.-B. Doc, C. Vergez, P. Guillemain, and J. Kergomard, "Sound production on a "coaxial saxophone"," The Journal of the Acoustical Society of America, vol. 140, no. 5, pp. 3917-3924, 2016.

[22] A. D. Pierce and P. Smith, Acoustics: An introduction to its physical principles and applications. Acoustical Society of America, 1981.

[23] H. Levine and J. Schwinger, "On the radiation of sound from an unflanged circular pipe," Physical review, vol. 73, no. 4, p. 383, 1948.

[24] F. Silva, P. Guillemain, J. Kergomard, B. Mallaroni, and A. N. Norris, "Approximation formulae for the acoustic radiation impedance of a cylindrical pipe," Journal of Sound and Vibration, vol. 322, no. 1-2, pp. 255-263, 2009. 
[25] R. H. Byrd, R. B. Schnabel, and G. A. Shultz, "A trust region algorithm for nonlinearly constrained optimization," SIAM Journal on $\mathrm{Nu}$ merical Analysis, vol. 24, no. 5, pp. 1152-1170, 1987.

[26] T. F. Coleman and Y. Li, "An interior trust region approach for nonlinear minimization subject to bounds," SIAM Journal on optimization, vol. 6, no. 2, pp. 418-445, 1996.

[27] J.-P. Dalmont, B. Gazengel, J. Gilbert, and J. Kergomard, "Some aspects of tuning and clean intonation in reed instruments," Applied acoustics, vol. 46, no. 1, pp. 19-60, 1995.

[28] E. Moers and J. Kergomard, "On the cutoff frequency of clarinet-like instruments. geometrical versus acoustical regularity," Acta Acustica united with Acustica, vol. 97, no. 6, pp. 984-996, 2011.

[29] J.-B. Doc and C. Vergez, "Oscillation regimes produced by an alto saxophone: Influence of the control parameters and the bore inharmonicity," The Journal of the Acoustical Society of America, vol. 137, no. 4, pp. 1756-1765, 2015.

[30] B. Ricaud, P. Guillemain, J. Kergomard, F. Silva, and C. Vergez, "Behavior of reed woodwind instruments around the oscillation threshold," Acta Acustica united with Acustica, vol. 95, no. 4, pp. 733-743, 2009. 